\title{
Dental arch symmetry analysis in orthodontic treatment
}

\author{
Irsan Kurniawan, Eky Setiawan Soeria Soemantri, Ida Ayu Evangelina \\ Department of Orthodontics Faculty of Dentistry Universitas Padjadjaran
}

\begin{abstract}
The aims of this study were to introduce fixed symmetrograph as a device to analyze arch symmetry and to compare the result of arch symmetry measurements using such appliance with commonly used symmetrograph in Faculty of Dentistry Padjadjaran Universitas (Orthocross). Twenty based study models used in this study. The method used in this study was by comparing the result of measurement using orthocross with the result of measurements using fixed symmetrograph. The result of this study revealed there was no significant difference between measurement using orthocross with measurement using fixed symmetrograph $(P>0,05)$. Based on the result of this study, it is recommended to use this fixed symmetrograph, because there were several advantages using this device, for example symmetrograph's midlines superimpose exactly with study model's midlines with the aid of two vertical pins. Even more, the study model can be fixated using two fixators so that the study model and fixed symmetrograph were not moving during measurement.
\end{abstract}

Key words: Symmetrograph, symmetry, study model

\section{INTRODUCTION}

Like other medical or dentistry departments, the diagnosis in orthodontic treatment requires information about the patient and his/her problems. One of the methods to attain patient information is by analyzing the study model of patient's arch.

The study model analysis is routinely performed in the clinic and it is an important and inseparable step of orthodontic treatment. Before doing the analysis, a heptagonal base of the study model is made with good accuracy and symmetry to prevent a wrong visual impression when measuring jaw and dental arch shape symmetry. Proper occlusion is mandatory and reproducibility of the model should be possible. ${ }^{1}$
There are several types of study model analysis that are normally used at the Faculty of Dentistry Universitas Padjadjaran, such as symmetry, ALD, Bolton, Howes, and Moyers analysis.

Symmetry analysis is necessary to observe whether there is a midline deviation, canine rotation, buccal segment crowding, mesially tipped premolar, and unilateral crossbite. Moreover, it is expected that the symmetry of the right and left dental arch can be achieved through orthodontic treatment. ${ }^{2,3}$

Some of the instruments that can be used to measure dental position symmetry are Schmuth symmetric grid, symmetric scope, Korkhaus symmetric plates, and Bernklau Symmetrograph. ${ }^{2}$ These instruments are rarely found in the market 
and are not used in the Orthodontics Clinics at the Faculty of Dentistry Universitas Padjadjaran. Therefore, the instrument used at the Faculty of Dentistry Universitas Padjadjaran is a $19.5 \times 18.0$ $\mathrm{cm}$ transparent plastic with $0.5 \mathrm{~mm}$ thickness made by a student of the Faculty of Dentistry Universitas Padjadjaran which is usually called orthocross.

The aim of this paper is to introduce a modification of symmetrograph appliance and to compare the result of arch symmetry measurement using orthocross and using the fixed symmetrograph.

\section{Study model analysis}

Study model analysis is a measurement of maxillary and mandibular dental arch and dental occlusal relationship in three dimensions. Certain correlation between arch length, arch width, and dental mesio-distal width can also be observed using this analysis. Many authors write it as an index such as Pont, Howes, Linder, Harth, and Korkhaus index which is usually used in Germany. In this model analysis, the real value of the patient is compared with the standard value of the "normal arch". With the newest knowledge, it is considered that this method has minimum diagnostic value, but however it is still used this method widely. ${ }^{2}$

Aside from those limitations, the big advantage of model analysis is that it can diagnose malocclusion degree in three planes of face. Study model is analyzed towards the midpalatal raphe area (mid-sagittal plane), transverse plane and horizontal plane (Fig.1). 2,4

\section{Dental arch symmetry analysis}

The transversal and sagittal dental arch symmetry of the study model is measured from the occlusal view. ${ }^{3}$ The symmetry analysis determines the dental position differences of the left and right dental arch in the transversal and sagittal orientation.

This measurement requires a proper reference. Midpalatal raphe or known as maxillary median line is determined using two anatomic points, which are the reference points for transversal symmetry analysis. It is rather difficult to determine the mandibular median line because the direct transfer of midpalatal raphe is inaccurate and the reference points of mandibular is not as clear as those in the maxillary median raphe. The anterior point of mandibular median line can be determined more accurately using a mental spine film or lingual fraenum. The posterior point that is used to decide the mandibular median line is determined by a perpendicular line; drawn from the midpalatal raphe posterior border of maxillary study model to the mandibular study model. ${ }^{2}$

The midpalatal raphe is made with the help of two anatomic reference points on the palatine raphe. The anterior point $(X)$ is an intersection between the second rugae palatine with the palatine raphe. The posterior point is a border between hard palatum and soft palatum of the raphe, approximately at the center of two foveapalati. $^{2}$

The result of symmetry analysis and Korkhaus analysis can provide difference comparison of dental arch symmetry and reference points in an orthodontic treatment procedure.

The asymmetry of transversal and sagittal plane is frequently associated with midline deviation, canine rotation, crowding, mesially tipped premolar, buccal or lingual unilateral crossbite. The total or partial asymmetry can be observed and localized quickly, like drifting, tipping, and dental rotation. . $^{2,3}$ This simple step is really useful for planning dental movement and for determining the orthodontic appliance design.

\section{The dental arch transversal symmetry analysis}

Midpalatal raphe (MPR) is a maxillary median line and a reference point for determining transversal symmetry. From this measurement, the symmetry between right and left dental arch can be determined and the harmony or dysharmony of dental midline and skeletal midline is possible to observe. $^{2}$

From the diagnostic point of view, the measurement of transversal symmetry has clinical relevance, especially in malocclusion cases with transversal orientation such as posterior crossbite, edge-to-edge occlusion, buccal or lingual nonocclusion. ${ }^{2}$

The above study model case example shows a transversal malocclusion on the right posterior region, that is a unilateral crossbite with approximately $1.5 \mathrm{~mm}$ mandibular midline deviation to the right. ${ }^{2}$ To find out the cause of 
this mal-relation, first the transversal symmetry should be analyzed to determine width asymmetry of right and left maxillary and mandibular dental



Figure 1. Orientation planes of the study model.

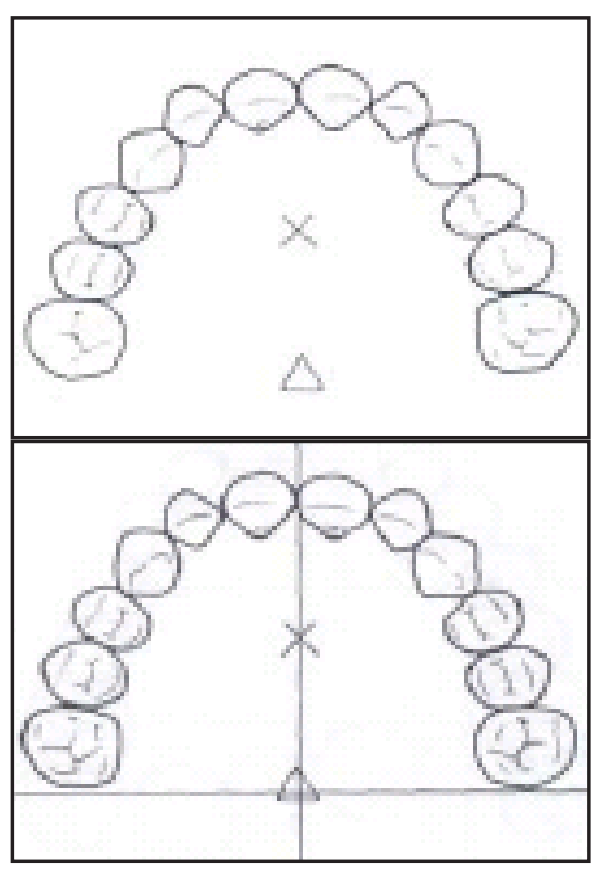

Figure 2. Determining the midpalatal raphe. ${ }^{2}$

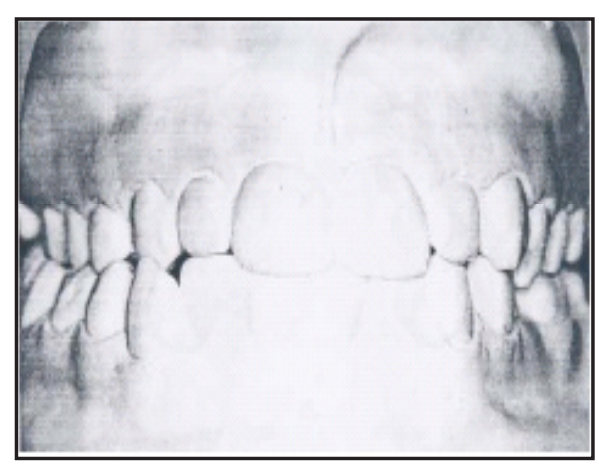

Figure 3. Unilateral crossbite of the right side. ${ }^{2}$ arches. $^{2}$

The analysis of maxillary dental arch shows symmetry of the dental arch. On the transversal

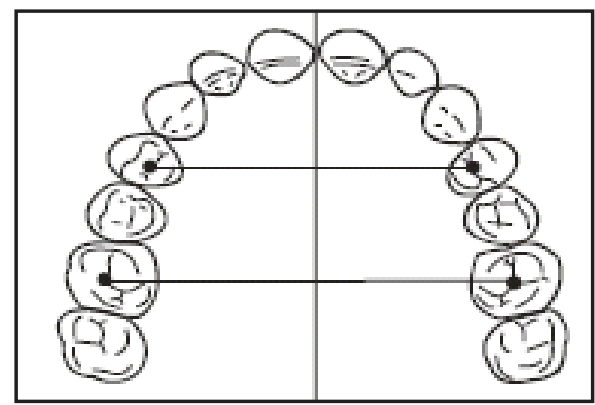

Figure 4. Symmetry maxillary dental arch.



Figure 5. Asymmetry mandibular dental arch.

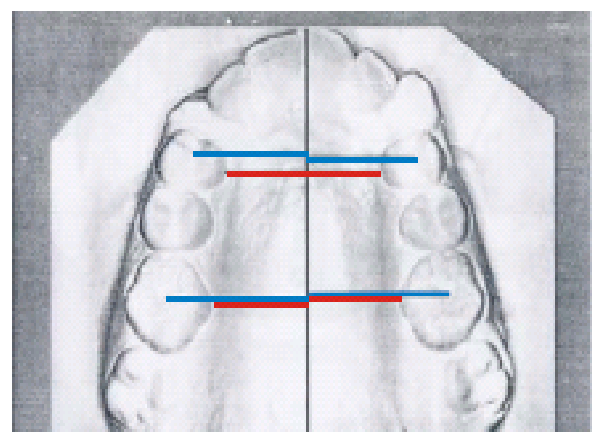

Figure 6. Sagittal symmetry analysis of a molar rotation. Blue line: normal case, red line: case of molar rotation.

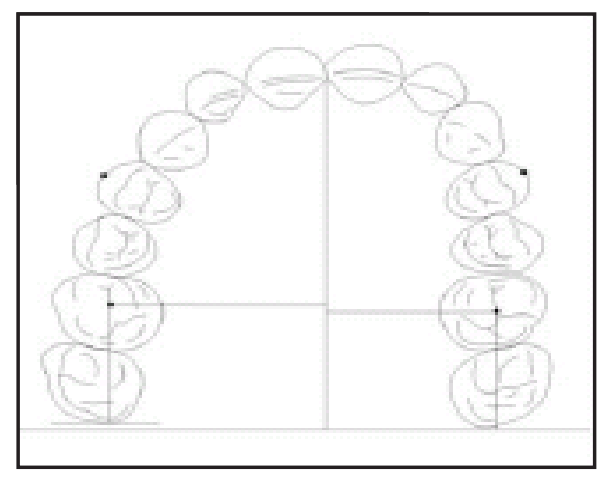

Figure 7. Correlation between transversal and sagittal asymmetry. ${ }^{2}$ 
symmetry analysis, the right and left dental arches have the same width in relation to midpalatal raphe. In this case, the transversal symmetry is determined using Pont reference points ${ }^{2}$, the distal pits on the occlusal surtaces of the first premolars and the central fossa of the first permanent molars. Mandibular arch analysis shows asymmetry development between the right side and the left side. The distance between right posterior teeth to the median line of the mandibula is wider than the other side. ${ }^{2}$

Based on this analysis, the unilateral crossbite showed in Fig. 3 is caused by the unilaterally wider mandibular right posterior dental arch..$^{2,3}$

\section{Dental arch sagittal symmetry analysis}

The sagittal symmetry is measured using the relationship between tuberosity plane that is perpendicular to the midpalatal raphe (MPR) and extended through the most distal maxillary tuberosity. This measurement can show the dental mesio-distal position asymmetry.

Practically, this measurement can be done by drawing a line that is parallel with the tuberosity area, drawn through the most posterior surface of the first molar, and by comparing the sagittal distance of the teeth (it is postulated that the first molar rarely migrates to the distal).

This analysis helps early diagnosis for each mesial dental movement. If the right and left sides are not asymmetric, it means that the dental right side and the left side are in a proper position or it means that there is a mesially symmetrical displacement.

In this case, the mesially displaced teeth is diagnosed by determining other criteria such as crowding and space loss, especially in the supporting zones; dental midline deviation in the presence of crowding and space loss; mesially tipped premolar; permanent first molar rotation; relationship between the first pair of rugae palatine to the canine based on Hauser (if the first rugae palatine is located distal from the canine, it means that there is a mesially displaced tooth); relationship of papilla-transversal area to the canine based on Schmuth (if the perpendicular line drawn through posterior tip of incisive papilla is located at the distal to the canine, it means that there is a mesially displaced tooth).
Sagittal symmetry in molar rotation case

Clinically the bilateral posterior tooth position to the mesial is similar to mesio-lingual rotation of a permanent first molar. In such first molar rotation case, sagittal symmetry analysis cannot be done using the Pont reference point. In this case, the mesial migration should be determined by measuring the mesio-lingual surface or disto-lingual of the permanent first molar. ${ }^{2}$

\section{Correlation between sagittal and transversal dental position}

As the result of dental arch width increment, the asymmetric dental arch in transversal orientation may make the more buccal dental position become relatively more mesial. ${ }^{2}$

When there is no mesial drifting of the posterior teeth, the position of the first molar can become asymmetric. This sagittal divergence is caused by the difference of right and left transversal arch form. ${ }^{2}$

\section{Dental arch symmetry measuring appliance}

Asymmetric dental arch form in transversal and sagittal orientation can be measured using the right measurement apparatus that is oriented on midpalatal raphe and tuberosity area. The dental arch is frequently measured using the Schmuth Symmetric Grid, Symmetric Scope, Korkhaus Symmetric Plates, and Bernkleu Symmetrograph. These appliances are rarely seen in the market and are not used in the Orthodontics Clinic at the Faculty of Dentistry, Universitas Padjadjaran. Therefore, the ruler grid used in the Faculty of Dentistry Universitas Padjadjaran is only a $19.5 x$ $18.0 \mathrm{~cm}$ transparent plastic with $0.5 \mathrm{~mm}$ thickness which was created by a student of the Faculty of Dentistry Universitas Padjadjaran and is commonly referred as orthocross.

The main problem that regularly appears at the Faculty of Dentistry University Padjadjaran is the inaccuracy of orthocross when it is used as a measurement tool. This usually occurs because while measuring, the orthocross position is not stable. The orthocross midline does not superimpose with the study model midline.

The inaccuracy in dental arch symmetry measurement, however, may occur due to the following reasons: The heptagonal base is not 


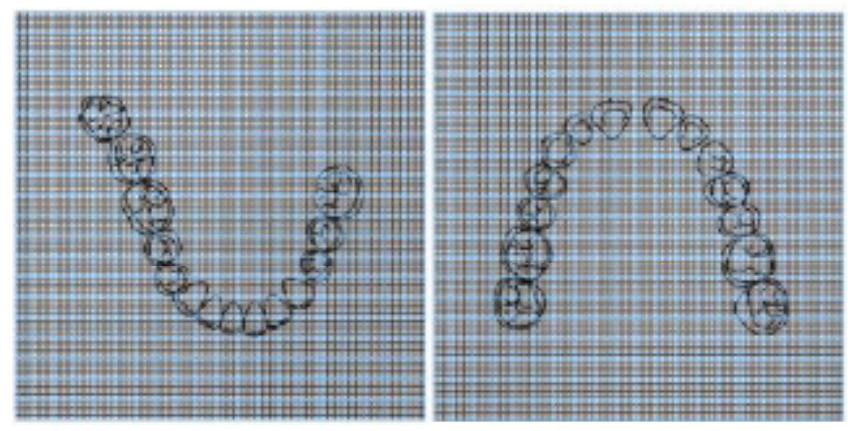

Figure 8. Symmetry analysis using Orthocross.

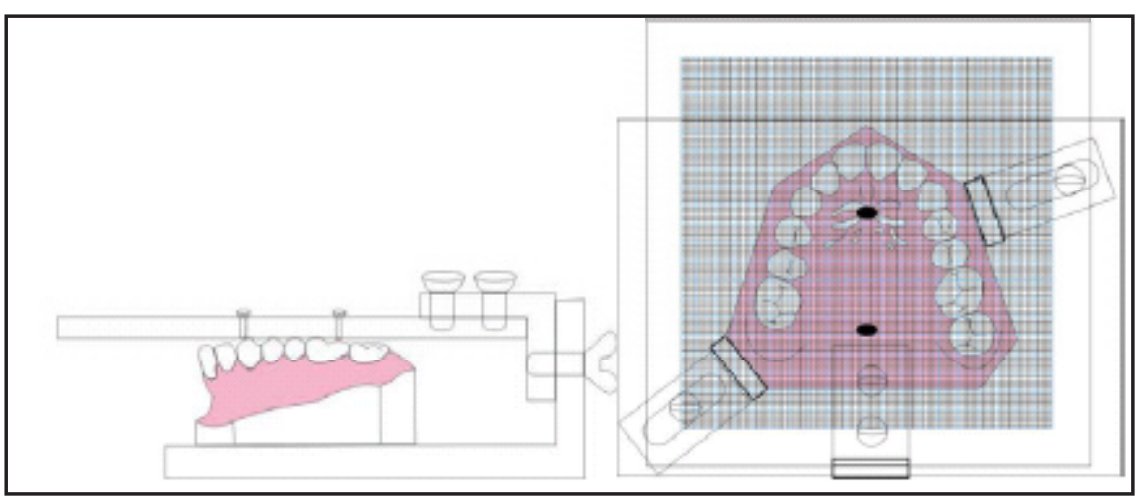

Figure 9. Left: Symmetry analysis using fixed symmetrograph with vertical pins to superimpose the appliance midline and study model midline. Right: Fixed symmetrograph with a study model fixator.

correctly made; The reference points for creating median line are not appropriately determined; The dental reference points are improperly determined; The appliance moves during the measurement; The study model moves during the measurement; and the viewpoint is not perpendicular with the appliance and study model.

Based on the above limitations, this paper will introduce a modification of symmetrograph appliance, called fixed symmetrograph that can minimize the orthocross measurement inaccuracy. The result of measurement using fixed symmetrograph is compared with the results of measurement using orthocross used at Faculty of Dentistry Universitas Padjadjaran.

\section{MATERIALS AND METHODS}

Twenty study model heptagonal bases were made and a midline from reference points on labial fraenum, intersection of second rugae palatine, and the center of fovea-palatini were drawn. ${ }^{2}$ ${ }^{5}$ The maxillary midline is then projected to the mandibular to create study model midline on mandibular. The patient study model used in the measurement had complete teeth starting from the right maxillary first molar until left maxillary first molar and from the left mandibular first molar until right mandibular first molar; there were no abnormality in the dental form and number.

After the sample criteria in this research were determined, a pilot study was held to calibrate an intra-examiner by measuring the same model twice. The symmetry of patient's mandibular and maxillary study models were then observed and measured in transversal and sagittal orientation by orienting the symmetrograph in a certain way until this appliance midline superimposed precisely on the study model midline and parallel with the occlusal surface. This can be more accurately done with fixed symmetrograph using two vertical pins on the symmetrograph midline that are projected directly on the study model midline.

First the orthocross in the Faculty of Dentistry Universitas Padjadjaran was used to measure the symmetry and it is followed by fixed symmetrograph. The results were documented and compared in millimeter scale. 


\section{RESULTS}

This research shows that there are no significant differences between the results of measurement using orthocross and fixed symmetrograph ( $p>0.05)$. This means that fixed symmetrograph is feasible to be used, just like the orthocross. However, there are advantages of fixed symmetrograph for measuring dental arch symmetry, for example there are 2 vertical pins that connect the symmetrograph midline and study model midline so the symmetrograph midline can superimpose the study model midline. This also supports the measuring accuracy. In addition, the measurement can be done faster.

Besides, fixed symmetrograph has a fixator so the study model can be fixed in one place and will not move and also it is not necessary to hold it while measuring. This helps to get an accurate and constant result if the measurement is done more than once. From the endurance point of view, fixed symmetrograph is made of acrylic with $1.5 \mathrm{~mm}$ thickness so it is more durable than orthocross, which is made of transparent plastic that is relatively easier to be broken.

\section{DISCUSSION}

The measurement was done according to the method that has been explained earlier, but there are some problems when measuring using orthocross, that is the difficulty to superimpose the orthocross midline with the model study midline.Besides, the orthocross position which is prone to move makes the measuring difficult.

This makes measuring dental arch symmetry using orthocross requires high concentration and longer time.

\section{CONCLUSION}

It can be concluded from this research that fixed symmetrograph can be used for measuring dental arch symmetry. This appliance has several advantages compared to orthocross, which is commonly used in the Faculty of Dentistry Universitas Padjadjaran, such as there are 2 vertical pins that connect fixed symmetrograph midline and study model midline so that fixed symmetrograph midlines superimposes exactly with study model midlines. There are also two fixators so the fixed symmetrograph will not move during measurement.

\section{REFERENCES}

1. Houston WJB. Orthodontic walther. $4^{\text {th }}$ ed. Jakarta: Hipokrates; 1990.

2. Rakosi T. Color atlas of dental medicine: Orthodontic diagnosis. New York: Thieme Medical Publishers Inc.; 1993. p. 213-7.

3. Loh P. Basic guide in orthodontic diagnosis. Manila: Fourways Printing Inc.; 1999. p. 1167,132-3.

4. Linden VD. Diagnosis and treatment planning in dentofacial orthopedics. London: Quintessence Publishing Co. Ltd.; 1987. p. 140-3.

5. Profit WR. Contemporary orthodontics. $3^{\text {rd }}$ ed. Philadelphia: CV Mosby Co.; 2000. p. 165-6.

6. Moyers RE. Handbook of orthodontics. $4^{\text {th }}$ ed. Chicago: Year Book Medical Publishers Inc.; 1988. p. 364-7. 